\title{
Severity of Depressive Symptoms and Volume of Superior Temporal Gyrus in People Who Visit a Memory Clinic Unaccompanied
}

\author{
Taiki Kambe ${ }^{a-c}$ Asako Yasuda ${ }^{a}$ Setsuo Kinoshita ${ }^{a} \quad$ Masahiro Shigeta $^{a}{ }^{d}$ \\ Toru Kinoshita ${ }^{a}$ \\ ${ }^{a}$ Nozomi Memory Clinic, Tokyo, Japan; ${ }^{b}$ Department of Neurology, Juntendo University \\ School of Medicine, Tokyo, Japan; 'Kodama Clinic, Tokyo, Japan; d Department of Psychiatry, \\ The Jikei University School of Medicine, Tokyo, Japan
}

Keywords
Depression · Dementia $\cdot$ Memory impairment $\cdot$ Magnetic resonance imaging $\cdot$ Brain volume .
Memory clinic

\section{Abstract}

Background/Aims: Depression and cognitive decline are reported to be interrelated. Depression of older adults with memory complaints who seek medical help have not been well documented. This study was carried out to test the hypothesis that a relatively high level of depressive symptoms associated with brain structure is characteristic of people who visited a memory clinic unaccompanied (UA). Method: We retrospectively compared Center for Epidemiologic Studies Depression Scale (CES-D, for evaluation of depressive symptoms) scores of UA subjects ( $n=21$ ) with those of people who were accompanied $(n=75)$. Within each groups, we further examined the association between brain morphology and the CES-D scores using FreeSurfer software. Results: We found that the relatively high CES-D scores of UA subjects were inversely associated with the normalized volumes of bilateral superior temporal gyrus (STG). Conclusion: Our results suggest that depressive symptoms of UA subjects demonstrated by the relatively high levels of CES-D scores were primary, because of the inverse association with the normalized volume of bilateral STG. Thus, focusing on the depressive symptoms may be a suitable approach to satisfy potential medical needs of UA subjects with or without memory impairment. 


\section{Introduction}

Early recognition and intervention of cognitive impairment and dementia are considered as desirable. Early diagnosis allows people with dementia to facilitate planning while they still have the cognitive capacity to make important decisions about their future care. They can obtain access to available drug and non-drug therapies only after receiving a diagnosis, which may improve their cognition and enhance their quality of life [1].

Regarding early diagnosis of dementia, depression is important as it is associated with cognitive decline, represents a risk factor for dementia, and is distinguished from cognitive decline. Depression masquerading as dementia is likely the most common differential diagnosis and should always be considered; however, they can coexist, and depression may precede dementia [2]. Late-onset depression can be associated with the development of dementia [3]. It is unclear whether late-onset depression is a risk factor for or an early symptom of dementia [4]. In addition, a recent report indicated that mild cognitive impairment is a risk factor for depression [5].

Older adults with memory complaints who seek medical help are more likely to have close relatives with dementia. They also consider their complaints as serious concerns with underlying biomedical reasons and do not hold negative views about their doctor $[6,7]$. This population includes people who will develop dementia in the future and "worried well" individuals who are more concerned but perform normally in memory and non-memory tests [8]. Additional insight into whether older adults with memory complaints seek medical help is important for population-based interventions likely to be successful in decreasing cognitive severity at diagnosis of dementia. Thus, a comprehensive overview of this population is useful for not only clinical interventions but also strategies to increase early diagnosis of dementia. This population presents a high level of depressive symptomatology compared to the healthy population [9].

Brain imaging has started to yield information about the existence of biological bases of depression. Patients with major depression were characterized by diffuse bilateral grey matter loss in ventrolateral and ventromedial frontal systems extending into temporal gyri compared to healthy controls [10]. Another study demonstrated reduced right lateral inferior fontal volumes in major depression disorder [11]. Associations between depression and brain morphology of older adults with memory complaints who seek medical help have not been well documented.

This study focused on individuals who visited a memory clinic unaccompanied (UA), obviously included in older adults with memory complaints who seek medical help. We hypothesized that a relatively high level of depressive symptoms is a characteristic of UA individuals, associated with the brain structure. To test this hypothesis, we compared the degree of depressive symptoms of UA individuals with that of accompanied (AC) individuals, and we further investigated the relationship between the degree of depressive symptoms and brain structure for each group.

\section{Materials and Methods}

\section{Participants}

Participants were consecutive outpatients aged $>65$ years who visited the Nozomi Memory Clinic, an open-access institution, for the first time between September 2015 and February 2016. We retrospectively investigated 21 UA subjects and 75 AC subjects who had obtained neuroimaging data. This study was approved by the Asai Dermatology Institutional Review Board. Using the public website and in-hospital posters, we disclosed the prescribed 
Kambe et al.: Severity of Depressive Symptoms and Volume of Superior Tempora Gyrus in People Who Visit a Memory Clinic Unaccompanied

information and gave the subjects or, where appropriate, their nearest relatives the possibility to refuse participation.

\section{Neuropsychological Tests}

Neuropsychological tests involved the Mini-Mental State Examination (MMSE) and the Center for Epidemiologic Studies Depression Scale (CES-D) for evaluation of depressive symptoms.

\section{Image Acquisition and Analysis}

The subjects underwent brain magnetic resonance imaging (MRI) scans using a 1.5-tesla Optima MR360 Advance (General Electric Healthcare, Milwaukee, WI, USA), with an 8-channel head coil. High-resolution T1-weighted images were acquired with a three-dimensional fast spoiled gradient-recalled echo $\left(\mathrm{TR}=10.7 \mathrm{~ms}\right.$, echo time $=4.3 \mathrm{~ms}$, flip angle $=15^{\circ}$, voxel size $=1.0 \times 1.0 \times 1.0 \mathrm{~mm}^{3}$ and matrix size $=256 \times 256$ ), yielding $120-150$ contiguous slices of 1.2 -mm thickness in the sagittal plane, which were used for surface-based analysis. For participants with a neuropsychological assessment, all scans were performed within 1 month from cognitive testing. We identified the volumes of regions of interest (ROI volumes) using FreeSurfer software (version 5.3, http://surfer.nmr.mgh.harvard.edu/). This processing includes motion correction, removal of non-brain tissue using a hybrid watershed/surface deformation procedure, automated Talairach transformation, segmentation of the subcortical white matter and deep gray matter volumetric structures, intensity normalization, tessellation of the gray matter white matter boundary, automated topology correction, and surface deformation following intensity gradients to optimally segment borders at the location where the greatest shift in intensity defines the transition to the other tissue class [12]. The ROI included six limbic system and subcortical structures (hippocampus, amygdala, thalamus, caudate nucleus, putamen, and globus pallidus) and 31 cortical regions based on the Desikan-Killiany-Tourville atlas [13]. Our clinic analyzes head MRIs using FreeSurfer software in routine practice, and additional analysis by FreeSurfer has not been done for this research. The reconstructed images were visually examined without knowledge of the subjects' identities. From 27 subjects in the UA group and 101 subjects in AC group who obtained scanned data, 6 subjects of the UA group and 26 subjects of the AC group were excluded because of segmentation errors. For normalization, each subject's ROI volumes were divided by the volume of their entire brain.

\section{Statistical Analysis}

We compared basic demographic characteristics between UA and AC individuals using a $t$ test or $\chi^{2}$ test as appropriate. Comparisons of MMSE and CES-D scores between the groups were examined using an analysis of covariance (ANCOVA). Within each groups, we further examined the correlation between the normalized ROI volumes and the CES-D scores. Multiple comparisons using the Bonferroni correction were done for each hemisphere.

\section{Results}

This study included 21 UA subjects and 75 AC subjects. Regarding the gender ratio, subject groups were similar $\left(\chi^{2}=1.329, p=0.2490\right)$ (Table 1$)$. Subjects in the UA group had significantly lower ages $(p<0.001)$, higher MMSE scores $(p<0.001$, ANCOVA controlling for age and sex), and higher CES-D scores ( $p=0.0054$, ANCOVA controlling for age, sex, and MMSE score) than those in the AC group. 
Kambe et al.: Severity of Depressive Symptoms and Volume of Superior Temporal

Gyrus in People Who Visit a Memory Clinic Unaccompanied

Table 1. Participant characteristics

\begin{tabular}{lccc}
\hline & $\begin{array}{l}\text { Unaccompanied } \\
(n=21)\end{array}$ & $\begin{array}{c}\text { Accompanied } \\
(n=75)\end{array}$ & $p$ value \\
\hline Age, years & $76.1(5.2)$ & $81.2(6.4)$ & $<0.001^{\mathrm{a}}$ \\
Females, $n$ & $12(57 \%)$ & $50(67 \%)$ & $\chi^{2}=1.329,0.2490^{\mathrm{b}}$ \\
MMSE score & $26.6(2.7)$ & $19.6(6.4)$ & $<0.001^{\mathrm{c}}$ \\
CES-D score & $13.4(10.6)$ & $8.9(6.1)$ & $0.0054^{\mathrm{d}}$ \\
\hline
\end{tabular}

Data are presented as mean (SD) or as stated. Characteristics were measured during the subjects' first visit to the clinic. MMSE, Mini-Mental State Examination; CES-D, Center for Epidemiologic Studies Depression

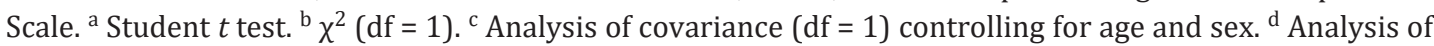
covariance $(\mathrm{df}=1)$ controlling for age, sex, and MMSE score.

ROI volumes of $21 \mathrm{UA}$ subjects and $75 \mathrm{AC}$ subjects were identified. In UA subjects, volumes of the right superior temporal gyrus (STG) were strongly associated with CES-D scores $(r=$ $-0.7234, p=0.0002$, remaining significant after Bonferroni correction) (Table 2). Volumes of left STG were only moderately associated with CES-D scores $(r=-0.6127, p=0.0032$, not significant after Bonferroni correction). Both associations were negative. No significant correlation was found between CES-D scores and normalized ROI volumes in AC subjects (data not shown).

\section{Discussion}

This study demonstrated that subjects in the UA group had higher CES-D scores than those in the AC group, and CES-D scores of UA subjects were inversely associated with the normalized volume of the right STG and only moderately associated with the left STG. There was no association between CES-D scores and normalized ROI volumes in AC subjects; this may have been due to clinical heterogeneity of AC subjects.

We focused on UA subjects as they chose our clinic to get their symptoms treated, made a reservation, and visited the clinic as scheduled. Hence, they had well-maintained executive functions and sought medical help on their own accord. Since our clinic's name contains "Memory," we are known as specializing in problems related to memory. Typically, individuals who visit our clinic on their own accord are self-aware of their memory impairment. Thus, UA subjects were included in our analysis of older adults with memory complaints seeking medical help.

The right STG is activated during theory of mind tasks [14]. This area is associated with attention to emotion [15] and face processing [16]. Decreased volume of the right STG is considered as a structural neural marker of social-emotional information evaluation abnormalities [17]. A volumetric study demonstrated reduction in the volume of the left STG in patients with anxiety disorders [11]. In addition, the CES-D scale evaluates both depression and anxiety $[18,19]$. Together with these findings, our results indicate that a relatively high level of depressive symptoms in UA subjects was caused by problems of emotion processing especially anxiety, considering the inverse association with STG volume. This may correspond to the findings that older adults with memory complaints seeking medical help are more likely to have a relatively high level of concern [7].

Our results also suggest that depressive symptoms of UA subjects demonstrated by the relatively high levels of CES-D scores were primary, because of the inverse association with 
Table 2. Correlations between CES-D scores and normalized ROI volumes in 21 subjects who visited the memory clinic unaccompanied

\begin{tabular}{|c|c|c|c|}
\hline & & \multicolumn{2}{|c|}{ Correlation coefficient ( $p$ value) } \\
\hline & & left & right \\
\hline \multirow[t]{7}{*}{ Temporal lobe } & Entorhinal & $-0.1259(0.5867)$ & $-0.2260(0.3247)$ \\
\hline & Parahippocampal & $-0.2572(0.2604)$ & $-0.05378(0.8169)$ \\
\hline & Fusiform & $-0.2772(0.2238)$ & $-0.1985(0.3883)$ \\
\hline & Superior temporal & $-0.6127(0.0032) *$ & $-0.7234(0.0002)^{*, a}$ \\
\hline & Middle temporal & $0.1872(0.4166)$ & $-0.4574(0.0371)$ \\
\hline & Inferior temporal & $-0.4080(0.0663)$ & $-0.1398(0.5455)$ \\
\hline & Transverse temporal & $-0.2081(0.3653)$ & $-0.1511(0.5133)$ \\
\hline \multirow[t]{10}{*}{ Frontal lobe } & Superior frontal & $-0.01974(0.9323)$ & $0.2542(0.2662)$ \\
\hline & Rostral middle frontal & $-0.04575(0.8439)$ & $-0.1818(0.4303)$ \\
\hline & Caudal middle frontal & $0.005405(0.9814)$ & $-0.1057(0.6485)$ \\
\hline & Pars opercularis & $-0.06774(0.7705)$ & $-0.1861(0.4192)$ \\
\hline & Pars triangularis & $-0.1007(0.6641)$ & $0.1325(0.5472)$ \\
\hline & Pars orbitalis & $0.07966(0.7314)$ & $-0.2382(0.2985)$ \\
\hline & Lateral orbitofrontal & $-0.3165(0.1622)$ & $-0.3312(0.1425)$ \\
\hline & Medial orbitofrontal & $-0.4063(0.0676)$ & $-0.1054(0.6493)$ \\
\hline & Precentral & $-0.2992(0.1877)$ & $-0.1713(0.4578)$ \\
\hline & Paracentral & $-0.07685(0.7406)$ & $-0.05988(0.7965)$ \\
\hline \multirow[t]{5}{*}{ Parietal lobe } & Postcentral & $0.1746(0.4491)$ & $0.04223(0.8558)$ \\
\hline & Supramarginal & $-0.3844(0.0853)$ & $-0.4493(0.0410)$ \\
\hline & Superior parietal & $-0.5369(0.0121)$ & $-0.1594(0.4901)$ \\
\hline & Inferior parietal & $0.2238(0.3294)$ & $-0.1172(0.6129)$ \\
\hline & Precuneus & $-0.04942(0.8315)$ & $-0.2067(0.3686)$ \\
\hline \multirow[t]{4}{*}{ Occipital lobe } & Lingual & $-0.1801(0.4347)$ & $-0.1524(0.5096)$ \\
\hline & Pericalcarine & $-0.1028(0.6574)$ & $-0.1175(0.6121)$ \\
\hline & Cuneus & $-0.02497(0.9145)$ & $0.04744(0.8382)$ \\
\hline & Lateral occipital & $0.1883(0.4138)$ & $-0.07079(0.7604)$ \\
\hline \multirow{5}{*}{$\begin{array}{l}\text { Cingulate cortex } \\
\text { and insula }\end{array}$} & Rostral anterior cingulate & $-0.3389(0.1329)$ & $-0.1245(0.5893)$ \\
\hline & Caudal anterior cingulate & $0.1381(0.5505)$ & $-0.1937(0.4001)$ \\
\hline & Posterior cingulate & $0.1440(0.5333)$ & $0.2519(0.2706)$ \\
\hline & Isthmus cingulate & $-0.2522(0.2700)$ & $-0.1213(0.6005)$ \\
\hline & Insula & $-0.1655(0.4374)$ & $-0.1355(0.5580)$ \\
\hline \multirow{3}{*}{$\begin{array}{l}\text { Limbic system } \\
\text { and thalamus }\end{array}$} & Hippocampus & $0.1819(0.4300)$ & $-0.03129(0.8929)$ \\
\hline & Amygdala & $0.1763(0.4447)$ & $-0.2156(0.3480)$ \\
\hline & Thalamus & $-0.1780(0.4401)$ & $-0.18875(0.4126)$ \\
\hline \multirow[t]{3}{*}{ Basal ganglia } & Putamen & $0.1375(0.5522)$ & $-0.1489(0.5194)$ \\
\hline & Caudate nucleus & $0.1088(0.6385)$ & $0.1023(0.6569)$ \\
\hline & Globus pallidus & $-0.05053(0.8278)$ & $-0.07744(0.7387)$ \\
\hline
\end{tabular}

${ }^{*} p<0.01 .{ }^{a}$ Significant after Bonferroni correction.

the normalized volume of bilateral STG. Thus, focusing on the depressive symptoms may be a suitable approach to satisfy potential medical needs of UA subjects with or without memory impairment. This may be supported by the findings that mild cognitive impairment is a risk factor for depression [5].

Importantly, the demographics of UA subjects vary owing to new medical developments and enlightenments on cognitive impairment. Sample size limitation, potential clinical hetero- 
Kambe et al.: Severity of Depressive Symptoms and Volume of Superior Temporal Gyrus in People Who Visit a Memory Clinic Unaccompanied

geneity of subjects, and a retrospective cross-sectional design of this study precluded definitive conclusions and did not allow for the generalization. Whether the negative correlation between CES-D scores and normalized volumes of STG in UA subjects was due to a disease remains unclear. Although our findings have limitations, the results indicated that memory clinics may play a role as the entrance to medical care both for people with depression as well as for people with dementia or at risk of dementia. Additional prospective studies are needed to confirm our findings.

\section{Acknowledgements}

We are grateful to Tomoko Honda, Satoko Aoyama, Daiki Tanaka, Sakiko Ogawa, Chieko Kondo, and Michiko Sunaga, the stuff of Nozomi Memory Clinic, Tokyo, for collecting the data. They did not receive compensation for their contributions.

\section{Disclosure Statement}

The authors have no conflicts of interest to disclose.

\section{Funding Sources}

This research did not receive any specific grant from funding agencies in the public, commercial, or not-for-profit sectors.

\section{References}

1 Prince M, Bryce R, Ferri C: World Alzheimer Report 2011: The Benefits of Early Diagnosis and Intervention. London, Alzheimer's Disease International, 2011.

2 Diniz BS, Butters MA, Albert SM, Dew MA, Reynolds CF 3rd: Late-life depression and risk of vascular dementia and Alzheimer's disease: systematic review and meta-analysis of community-based cohort studies. $\mathrm{Br} \mathrm{J}$ Psychiatry 2013;202:329-335.

3 Robinson L, Tang E, Taylor JP: Dementia: timely diagnosis and early intervention. BMJ 2015;350:h3029.

4 Mitchell AJ: Depression as a risk factor for later dementia: robust relationship? Age Ageing 2005;34:207-209.

5 Mirza SS, Ikram MA, Bos D, Mihaescu R, Hofman A, Tiemeier H: Mild cognitive impairment and risk of depression and anxiety: a population-based study. Alzheimers Dement 2017;13:130-139.

-6 Hurt CS, Burns A, Brown RG, Barrowclough C: Why don't older adults with subjective memory complaints seek help? Int J Geriatr Psychiatry 2012;27:394-400.

7 Begum A, Whitley R, Banerjee S, Matthews D, Stewart R, Morgan C: Help-seeking response to subjective memory complaints in older adults: toward a conceptual model. Gerontologist 2013;53:462-473.

-8 Alladi S, Arnold R, Mitchell J, Nestor PJ, Hodges JR: Mild cognitive impairment: applicability of research criteria in a memory clinic and characterization of cognitive profile. Psychol Med 2006;36:507-515.

-9 Ramakers IH, Visser PJ, Bittermann AJ, Ponds RW, van Boxtel MP, Verhey FR: Characteristics of help-seeking behavior in subjects with subjective memory complaints at memory clinic: a case-control study. Int J Geriatr Psychiatry 2009;24:190-196.

10 Arnone D, Job D, Selevaraj S, Abe O, Amico F, Cheng Y, Colloby SJ, O’Brien JT, Frodl T, Gotlib IH, Ham BJ, Kim MJ, Koolschijn PC, Perico CA, Salvadore G, Thomas AJ, Van Tol MJ, van der Wee NJ, Veltman DJ, Wagner G, Mclntoch AM: Computational meta-analysis of statistical parametric maps in major depression. Hum Brain Mapp 2016;37:1393-1404.

11 van Tol MJ, van der Wee NJ, van den Heurvel OA, Nielen MM, Demenescu LR, Aleman A, Renken R, van Buchem MA, Zitman FG, Veltman DJ: Regional brain volume in depression and anxiety disorders. Arch Gen Psychiatry 2010;67:1002-1011.

$\$ 12$ Fischl B: FreeSurfer. Neuroimage 2012;62:774-781. 
13 Klein A, Tourville J: 101 labeled brain images and a consistent human cortical labeling protocol. Front Neurosci 2012;6:17171.

14 Vollm BA, Taylor AN, Richardson P, Corcoran R, Stirling J, McKie S, Deakin JF, Elliot R: Neuronal correlates of theory of mind and empathy: a functional magnetic resonance imaging study in a nonverbal task. Neuroimage 2006;29:90-98.

15 Narumoto J, Okada T, Sadato N, Fukui K, Yonekura Y: Attention to emotion modulates fMRI activity in human right superior temporal sulcus. Brain Res Cogn Brain Res 2001;12:225-231.

16 Morris JS, Friston KJ, Buchel C, Frith CD, Young AW, Calder AJ, Dolan RJ: A neuromodulatory role for the human amygdala in processing emotional facial expressions. Brain 1998;121:47-57.

17 Pan LA, Ramos L, Segreti A, Brent DA, Phillips ML: Right superior temporal gyrus volume in adolescents with a history of suicide attempt. Br J Psychiatry 2015;206:339-340.

18 Breslau N: Depressive symptoms, major depression, and generalized anxiety: a comparison of self-reports on CES-D and results from diagnostic interviews. Psychiatry Res 1985;15:219-229.

19 Dozeman E, van Schaik DJ, van Marwijk HW, Stek ML, van der Horst HE, Beekman AT: The center for epidemiological studies depression scale (CES-D) is an adequate screening instrument for depressive and anxiety disorders in a very old population living in residential homes. Int J Geriatr Psychiatry 2011;26:239-246. 